\title{
DAs PotPourRI ALS KomplementärerscheinUng ZUM KANON BÜRGERLICHER KUNST UND ZEICHEN DER MODERNE
}

\author{
Sabine Giesbrecht
}

Unter der Überschrift Musikalische Edelsteine sind in den Jahren 1908 bis $1939^{1}$ im Abstand von ein bis zwei Jahren 17 Bände mit Salonmusik für Klavier oder Klavier und Gesang erschienen, die den Gegenstand der folgenden Untersuchung bilden. Der Titel suggeriert, dass es sich um ein hochwertiges Repertoire handelt. Schlägt man das Inhaltsverzeichnis auf, so wird dagegen klar, dass nur wenige Lieder, Arien und Salonstücke künstlerischen Ansprüchen nahe kommen. Es überwiegen Couplets aus Operetten, Revuen und Possen, Potpourris sowie Modetänze und Stücke, die eher ein Leben an der Peripherie oder jenseits des offiziellen Kunstkanons führen. Das deutet der Untertitel an, in dem die Begriffe »modern und beliebt « sowie »ernst und heiter ${ }^{2}$ jene Mischung aus Bildung und Unterhaltung ankündigen, mit der unterschiedliche Interessenten angesprochen werden können. In dieser Vielfalt und der damit verbundenen Tendenz zur Gleichrangigkeit von U- und EMusik zeigen sich Elemente einer Moderne, die sich den Normen der Kunst noch verpflichtet weiß, aber herrschenden ästhetischen Leitideen immer mehr zu entziehen sucht.

Potpourris ${ }^{3}$ sind Prototypen dieser Entwicklung, da sie anpassungsfähig sind und sich thematisch nach allen Seiten hin zu öffnen vermögen. Als Opern-, Komponisten- oder Stil-Potpourris stehen sie anerkannten Kunst-

1 Das Erscheinungsdatum der 17 Bände Musikalische Edelsteine ist nirgends angegeben. Die Angaben zur Datierung richten sich nach dem jeweils letzten Copyright-Vermerk.

2 Der Inhalt bleibt in allen Bänden etwa gleich: »Opern- und Operetten-Musik «, "Salon- und Tanzmusik«, "Heitere und ernste Lieder «. Band I (1908) enthält zusätzlich »Gelegenheitsmusik « und »Humoristika«. In Band XIII (1930) kommen »Märsche«, in XVI (1937) der »Tonfilm« und in XVII (1939) »Volksmusik« als Rubriken hinzu.

3 Es wurden nur Stücke ausgewählt, die den Begriff »Potpourri« oder »Fantasie über...« explizit im Titel führen. 
formen nahe, während das Schlager-Potpourri sich auf Operettenmelodien, Gesellschaftstänze oder andere Arten unterhaltender Musik stützt. Die Kontinuität ihres Erscheinens gibt zu Überlegungen Anlass, warum gerade dieses Genre sich erfolgreich behaupten konnte.

Das Prestige dieser Musik ist mäßig bis mangelhaft. Opern- und Komponisten-Potpourris werden von Musikkennern vielfach als Billigform und Substitut für Ungebildete (Adorno 1964: 22) diffamiert oder als Angriff auf die Authentizität bedeutender Werke betrachtet. Die Abhängigkeit eines Potpourris von der Kunst wertet es aus Sicht der Kenner nicht auf, sondern zieht den Vergleich mit dem Original und seinen ästhetischen Normen nach sich. ${ }^{4}$ Diese sind das Substrat musikalischer Spitzenleistungen und gehören zu einem Kanon von Gattungen und Werken, die als bildend, zeitlos gültig, individuell, vorbildlich und identitätsstiftend gelten.

Alles das ist das Potpourri nicht. Es kennt keine Komponisten, sondern Bearbeiter; es ist nicht einmal eine Komposition im herkömmlichen Sinne, obwohl viele Arrangeure das offenbar anders sehen und ihre Stücke selbstbewusst mit einer Opus-Zahl überschreiben. Die ungeordnete Reihungsform widerspricht dem traditionellen Werkcharakter, und die Anleihen bei einem fremden Repertoire gehen zu Lasten individueller Formulierungen. Das Potpourri ist kurzlebig, in der Regel leicht spielbar und auch dann noch unterhaltend, wenn es mit Anklängen aus der Bildungsmusik operiert.

Was Kennern als kritikwürdig erscheint, der »Diebstahl« musikalischer Gedanken, ist für das Potpouri konstitutiv. Als Ansammlung von Themen führt es ein Eigenleben, weist aber über sich hinaus auf erfolgreiche Neuerscheinungen oder »Evergreens « des Musiklebens. Diese werden jedoch nur in Häppchen dargereicht und sollen die ausführenden Musikerinnen und Musiker dazu animieren, bei Gelegenheit die Musik in vollem Umfang und im dazugehörigen sozialen Umfeld wahrzunehmen. Sie sind Erinnerungen an Erlebtes oder ein Vorgeschmack auf das öffentliche Repertoire und regen zum Besuch von Bühnenwerken, Filmen, Konzerten und anderen Musikveranstaltungen an. Damit nimmt das Potpourri strukturell und sozial eine Vermittlerstellung ein und ist nicht zu Unrecht als »mittlere Musik« bezeichnet worden (Dahlhaus 1972). Flankiert von den Kategorien Oben und Unten oder Hoch und Niedrig ist es auch im ästhetischen Wertesystem komplementär verbunden mit der bürgerlichen Kunst wie auch mit dem, was diese aus ihrem Kreis auszuschließen wünscht.

4 Robert Schumann (1889: 28) moniert 1836 »das Honigaussaugen an Bellinis Opern«. - Die Kritik am Schubert-Potpourri Dreimäderlhaus lebt vom entwertenden Vergleich. Die Bearbeiter werden als »rücksichtslose Ausbeuter « und »Geistesverbrecher « bezeichnet (zit. n. Hilmar/Jestremsky 1997: 69). 


\section{Verschiedene Formen}

Gemeinsam ist allen Potpourris die Aneinanderreihung bekannter Melodien, Themen oder geschlossener Abschnitte, ein Verfahren, mit dem die Vereinzelung von Werkteilen zum Herstellungsprinzip gemacht wird. Gehören die Zitate zu Opern, Operetten, Possen und Instrumentalmusik, so geben sie einen gewissen Überblick über das Ausgangsstück, im besten Fall Kurzfassungen davon wieder. Bei unzusammenhängenden Einzelstücken, zum Beispiel Tänzen, Volksliedern oder Schlagern, haben Überschriften die Aufgabe der Integration. Titel wie Berlin wackelt (IV, 1913), Deutsches VolksliederPotpourri (XI, 1927), Marschlieder-Potpourri (XIII, 1930) oder Das neue Schlagerpotpourri (XIV, 1931) übernehmen diese Rolle, kündigen aber lediglich die zu erwartende musikalische Form oder Gattung an, mit deren Material das Potpourri ausgestattet ist.

Umfang und Abfolge der einzelnen Melodien sind vollständig in das Belieben des Bearbeiters gestellt, der mit Hilfe selbst verfasster Übergänge, Eröffnungs- und Schlusspassagen ein in sich zusammenhängendes Stück herzustellen sucht. Die Herauslösung von Themen und Abschnitten aus ihrem ursprünglichen Kontext sowie ihre Bearbeitung und Neuplatzierung, auch im Potpourri, sind übliche Verfahren, die bei der Komposition populärer Bühnenwerke bereits im Voraus eingeplant werden. Bravourstücke und Erfolg versprechende Nummern aus Opern und Operetten sind oft schon während des Kompositionsaktes auf ihre isolierte Präsentation außerhalb der Werkgrenzen hin angelegt, um später als selbständige "Schlager (Geuen 1995: 60) Kasse zu machen. Im Potpourri sind sie noch einmal ökonomisch auf engstem Raum zusammengedrängt.

Die Zitate aus dem Bereich der Kunstmusik sind besonders kontextabhängig und büßen bei diesem Verfahren ihre energetischen Kräfte mehr oder weniger ein. Für das Komponisten-Potpourri der Edelsteine ${ }^{5}$ stehen dem Bearbeiter sämtliche Werke populärer Künstler zur Verfügung und sind im Potpourri-Arrangement in der Regel angegeben. Die nahezu unbegrenzte Auswahl und die Herkunft der Fragmente aus verschiedenartigen Formen und Gattungen führen zu einem relativ beziehungslosen Nebeneinander austauschbarer Einzelteile. Dennoch bleibt die Affinität zum Kunstwerk erhalten, die den Komponisten-Potpourris einen ästhetischen und sozialen Sonderstatus garantiert. Liebhaber dieser Musik können sich der Illusion hin-

5 Erinnerungen an Jacques Offenbach (II, 1910), „Wagneriana, Kleine Fantasie über die schönsten Motive aus Richard Wagners Opern« (V, 1914), Blütenkranz über Schuberts Melodien (VI, 1916). 
geben, ihr klassisches Repertoire erweitert zu haben und dem Ideal bürgerlicher Bildung ein Stück näher gekommen zu sein

An das »klassische " Repertoire angelehnt sind auch Opern-Potpourris, die mit Ausnahme von Band I durchgängig in der Reihe Musikalische Edelsteine auftauchen. ${ }^{6}$ Sie enthalten überwiegend Ausschnitte aus zeitgenössischen Bühnenwerken wie Madame Butterfly und Tiefland, die um 1916 (Band VI) noch den Reiz der Neuheit hatten. Das »modernste OpernPotpourri bezieht sich auf Schwanda, der Dudelsackpfeifer von Jaromir Weinberger, eine Oper, die bereits kurz nach der 1928 erfolgten Uraufführung in Band XIV (1931) der Edelsteine abgedruckt ist. Ältere Opern, wie Martha von Friedrich von Flotow (1847 komponiert, als Potpourri veröffentlicht in IV, 1913), werden offensichtlich wegen gängiger Arien auch in späteren Jahren noch als Potpourri vermarktet. Zum Standard-Repertoire gehören ebenfalls die Verdi-Opern La Traviata, Aida, Macht des Schicksals und Troubadour. Im Durchschnitt erscheinen die Opern-Potpourris etwa 20-40 Jahre nach der jeweiligen Ur- oder Erstaufführung.

Titel und Verfasser sind zur Orientierung angegeben. Eröffnet wird mit der Ouvertüre, der beliebte Arien, Ensemblestücke, Rezitative und Tänze folgen, allerdings nicht in vollständiger Fassung und nicht immer in einer dem Handlungsablauf entsprechenden Folge. Die Akteure der jeweiligen Oper werden genannt und dem unterlegten Libretto-Text zugeordnet. Dadurch ist ein kursorischer Überblick über das gesamte Werk möglich und eine gewisse Nähe zum Klavierauszug gegeben.

Operetten-Potpourris ${ }^{7}$ sind in den Edelsteinen etwa in gleicher Anzahl vertreten wie Opern-Potpourris. Einige aus diesem Metier stammende Schla-

6 Eugen d' Albert: Tiefland, 1903 (VI, 1916); Gaetano Donizetti: Don Pasquale, 1843 (XII, 1929); Friedrich v. Flotow: Martha, 1847 (IV,1913); Charles Gounod, 1859 u. 1869: Faust (III, 1912); Engelbert Humperdinck: Die Königskinder, 1897 u. 1910 und Hänsel und Gretel, 1893 (XIII, 1930 und XI, 1927); Wilhelm Kienzl: Der Evangelimann, 1895 (III, 1912); Leoncavallo: Bajazzo, 1892, Potpourri I u. II (VII, 1917 und VIII, 1920); Pietro Mascagni: Cavalleria rusticana, 1890 (IV,1913); Modest Mussorgsky: Boris Godunow, 1874 (X, 1925); Giacomo Puccini: Tosca, 1900 (X, 1925), Die Bohème,1896 (IX, 1922), Madame Butterfly, 1904 (VI, 1916); Peter I. Tschaikowsky: Eugen Onegin, 1879 (VIII, 1920, IX, 1922) u. Pique-Dame, 1890 (IX, 1922); Camille Saint-Saens: Samson und Dalila, $1877 \mathrm{u.}$ 1892 (XIII, 1930); Bedrich Smetana: Die verkaufte Braut, 1866 (IV, 1913); Giuseppe Verdi: La Traviata,1853 (II, 1910), Aida, 1871 (XI, 1927), Die Macht des Schicksals, 1861 (XV, 1936), Der Troubadour, 1853 (XII, 1929); Jaromir Weinberger: Schwanda, der Dudelsackpfeifer, 1928 (XIV, 1931); Ermanno WolfFerrari: Fantasie: Der Schmuck der Madonna, 1911 und 1933 (XVI, 1937).

7 Ralph Benatzky: Im weißen Rössl, 1930 (XV, 1936); Jean Gilbert: Puppchen, 1912 (IV, 1913); Hermann Götz Der Widerspenstigen Zähmung, 1874 (VIII, 1920); Richard Heuberger: Der Opernball, 1898 (XVI, 1937); Sidney Jones: Die Geisha, 1896 (XII, 1929); Emmerich Kálmán: Gräfin Mariza,1924 u. Die Zirkus- 
ger werden im selben Band sowohl als Einzelstücke, wie auch im PotpourriArrangement dargeboten. Die Favoriten dieser Jahre sind Edmund Eysler, Leo Fall, Jean Gilbert, Emmerich Kálmán, Franz Lehár, Jacques Offenbach, Robert Stolz, Johann Strauss (Sohn) und Carl Zeller. Als Arrangeure sind vor allem Leopold Weninger, C. [amillo] Morena, Otto Lindemann, Oscar Fetrás und Gustav Groschwitz genannt.

Schlager verschiedener Herkunft verbergen sich unter dem Begriff »gemischte Potpourris«. Sie präsentieren in bunter Folge eine Zusammenstellung »erfolgreicher« (XV, 1936) und »moderner« (XIV, 1931) Melodiefolgen und können als besonders attraktives Warenangebot frisch vom Musikmarkt verstanden werden. Sie bestehen aus bis zu 21 verschiedenen Schlagern, die durch eine Einleitung, Zwischenspiele und eine Schlusspassage zu einem geschlossenen Spielstück ergänzt werden. Legt man die Serie Musikalische Edelsteine zugrunde, so sind sie pianistisch weniger anspruchsvoll als Opernoder Komponisten-Potpourris.

Das herrschende Reihungs-Prinzip scheint das eigentliche Skandalon gewesen zu sein, an dem sich die Entwertung des Potpourris in erster Linie festgemacht hat. Noch im Jahr 1962 finden sich im Artikel »Potpourri« der Musikenzyklopädie Die Musik in Geschichte und Gegenwart dafür Begriffe wie »Ragout « und »Zusammengekochtes « (Engel 1962) ${ }^{8}$, die verraten, dass es sich um grobe Kost für Rezipienten mit nur geringfügigen kulturellen Kompetenzen handelt, die den geistigen Rang der Kunst verkennen und nicht zu würdigen wissen. Ungeachtet seines zweifelhaften Rufes bei Kunstkennern ist jedoch das Potpourri zu einem äußerst beliebten Teil der bürgerlichen Hausmusik geworden, was die 17 Bände Musikalische Edelsteine unübersehbar belegen.

prinzessin, 1926 (X, 1925 u. XI, 1927); Martin Knopf: Die kleine Hoheit (VIII, 1920); Franz Lehár: Schön ist die Welt, 1931 u. Das Land des Lächelns, 1929 (XIV,1931 u. XIII, 1930); Jacques Offenbach: Hoffmanns Erzählungen, 1881 (II, 1910), Pariser Leben, 1866 (V, 1914), Die Großherzogin von Gerolstein, 1867 (VII, 1917), Die schöne Helena, 1864 (VI, 1916), Blaubart, 1869 (XII, 1929); Johann Strauss: Die Fledermaus, 1847 (XV, 1936), Der Zigeunerbaron, 1885 (XIII, 1930); Kurt Weill: Dreigroschenoper, 1928 (XIV, 1931); Robert Winterberg: Hoheit - Der Franz!, 1913 (V, 1914); Ermanno Wolf-Ferrari: Die neugierigen Frauen, 1903 (XVII, 1939); Carl Zeller: Der Vogelhändler, 1891 (X, 1925), Der Obersteiger, 1893 (XIV, 1931).

8 In der zweiten Auflage der MGG geht der Artikel-Verfasser distanzierter mit dem Arsenal der Entwertungen um (Ballstedt 1997). 


\section{Das Potpourri als Haus- und Salonmusik}

Die Hausmusik ist ein wichtiger Bestandteil der gesamten bürgerlichen Musikkultur und Basis des öffentlichen Musiklebens. Sie lebt vom Interesse und von den Fertigkeiten musikalischer Laien und ist auf ein Repertoire angewiesen, das den Kenntnissen und Fähigkeiten nicht-professioneller Musikliebhaber angemessen und zu gepflegter Unterhaltung geeignet ist. Als Klavier- oder Kammermusik erlaubt sie unbegrenzte Wiederholungen und ermöglicht die Aneignung verschiedenartiger Repertoires. Dazu gehören spieltechnisch einfache oder mittelschwere, mäßig lange Stücke aus der Welt der »Klassik« ebenso wie unterhaltende Neukompositionen und Bearbeitungen, darunter Potpourris aller Art.

Glaubt man den Titelbildern der Reihe Musikalische Edelsteine (Band V-VIII, XI-XVI), so versammeln sich Familienmitglieder oder Freunde am Klavier, um sich miteinander zu unterhalten, die musikalischen Darbietungen zu genießen oder vielleicht auch zu hören, was es Neues in der Musikwelt gibt. Die Abbildungen zeigen außerdem ein bürgerliches Umfeld, den gemütlichen Salon mit dem Klavier im Zentrum, das in Mittelstandskreisen zur Standardausstattung gehört, sowie gut gekleidete, interessiert lauschende Zuhörerinnen und Zuhörer. Offensichtlich geben gesellige Zusammenkünfte dieser Art dem Familienleben nicht zu unterschätzende Impulse.

Das Potpourri fügt sich in diesen sozialen Rahmen perfekt ein, da es in seinen verschiedenen Formen leichte bis mittelschwere Klavier- und Kammermusik zu Bildungs- und Unterhaltungszwecken anbietet. Befürworter sind im bürgerlichen Lager die Volkserzieher, welche zumindest einigen Arten des Potpourris den pädagogischen Nutzen nicht absprechen möchten. So verweist zum Beispiel Karl Storck (1911: 102) auf den Wert des »künstlerischen« Potpourris mit Ausschnitten aus Opern oder als Zusammenstellung von Volksliedern. Wer in der Provinz ohne Musiktheater und Konzertaufführungen lebt oder sich einen Theaterbesuch nicht immer leisten kann, hat die Möglichkeit, damit etwas über Kunst zu erfahren. Eine Oper ließe sich in Ansätzen auch durch ein Potpourri kennen lernen, wenn die Zitate in einer sinnvollen Reihenfolge wiedergegeben sind und dadurch der Inhalt eines Werkes verständlich wird. Lieder-Potpourris mit Beiträgen zum berufsständischen Leben von Studenten, Jägern oder Soldaten führen seiner Meinung nach ebenfalls auf lebendige Art in die Welt der Kunst ein. Entscheidend sei, einem einfachen Publikum ein gewisses Maß an Bildung zu bieten; ästhetische Abstriche müssten dafür in Kauf genommen werden. 
Diese Auffassung macht deutlich, dass eine Trennung von Bildungs- und Unterhaltungsmusik im Bewusstsein zwar vorhanden, aber in lebenspraktischen Zusammenhängen nicht durchführbar ist. In ästhetischer Hinsicht streben Kunst und Unterhaltungsmusik auseinander, im sozialen Leben können sie nicht ohne einander auskommen (Giesbrecht 2000).

Erst gemeinsam bilden sie die Gesamtheit des Musiklebens, in dem die traditionelle Kunst wie auch Formen der modernen Unterhaltungsmusik einander ergänzen.

\section{Ein Schubert-Potpourri}

Das Bemühen, Klassisches, oder das, was man dafür hielt, unter die Leute zu bringen, zeigt sich vor allem an den Komponisten-Potpourris. Unter der Rubrik »Salon- und Tanzmusik« im Band von 1916, mitten im Ersten Weltkrieg und im Erscheinungsjahr des Singspiels Dreimäderlhaus, das Potpourri Blütenkranz über Schuberts Melodien - ausnahmsweise ohne Hinweis auf den Arrangeur. Es zeigt, wie nahe das Stück einerseits an das kanonisierte Repertoire heran reicht, andererseits aber herrschenden Kunstnormen keineswegs entspricht.

Die Form ergibt sich nicht aus einem kompositorischen Konzept, sondern beruht auf der willkürlichen Auswahl von Themen. Um von ihrem Genuss nicht abzulenken, sind Überleitungen eingefügt, die vorhandene thematische Gegensätze abmildern und das jeweils Neue vorbereiten. Zum Schluss wird dem Hörer mit Hilfe aufwändiger und geradezu aufdringlicher Kadenzbildungen überzeugend mitgeteilt, dass nun keine weiteren Zitate mehr zu erwarten sind.

Der bunte Blütenkranz ist mit korrespondierenden Fanfarenklängen eingerahmt, die beide aus dem Militärmarsch D 733, Nr. 1, stammen. Sie wirken als Klammer für die im Folgenden aufgeführten Schubert-Zitate:

1. Eröffnung: vierhändiger Militärmarsch Op. 51, Nr. 1 (D 733), ohne Angabe der Herkunft.

2. Teile der Ballettmusik zu Rosamunde (D 797).

3. Sehnsuchtswalzer, Nr. 2 aus der vierhändigen Walzersammlung, Op. 9.

4. Hauptteil des vierhändigen Militärmarsches Op. 51, Nr. 3 (D 733).

5. Nr. 7 aus Deutsche Tänze und Ecossaisen, vierhändig, Op. 33 (D 783).

6. Abschnitte aus Nummer 3 der Moments musicaux für Klavier, Op. 94 (D 780). 
7. Seitenthema des 1. Satzes aus der Sinfonie Nr. 8, der »Unvollendeten« (D 759).

8. Erste Strophe des klavierbegleiteten Sololiedes Ungeduld aus dem Zyklus Die schöne Müllerin mit Text von Wilhelm Müller.

9. Trio des unter 4. aufgeführten vierhändigen Militärmarsches Op. 51, Nr. 3 (D 733).

10. Schluss: vierhändiger Militärmarsch, Op. 51, Nr. 1 (D 733), ohne Angabe der Herkunft.

Grundprinzip ist die Abwechslung durch Themen und Abschnitte von unterschiedlicher Länge, die sich Spielern und Hörern als charakteristisch für das Werk Schuberts einprägen und das Interesse über das ganze Stück hin wach halten sollen. Die bekannte Ballettmusik zu Rosamunde ist mit ihren 84 (von insgesamt 283) Takten in eindrucksvoller Breite wiedergegeben. Es folgen Beispiele aus anderen musikalischen Gattungen, aus vier- und zweihändigen Klavierwerken, einem weiteren Orchesterwerk und einem klavierbegleiteten Sololied. Mit Ausnahme der Bühnenmusik und des Sinfonie-Themas handelt es sich um Beispiele geselliger Musik aus dem Umfeld von Schubertiaden, die in der bürgerlichen Hausmusik als kleine, feine Kunstformen wohl gelitten sind und auch in reduzierter Form innerhalb des Potpourris ihren ästhetischen Anspruch nicht ohne Weiteres aufgegeben haben.

Umso schwieriger ist es einer musikbegeisterten Öffentlichkeit zu vermitteln, warum Bearbeitungen von Kompositionen Schuberts nicht als Kunst zu betrachten sind. Größe und Bedeutung dieses Komponisten leiden gewiss nicht darunter, wenn charakteristische Teile aus dem Gesamtwerk extrahiert und als Keimzellen der Kunst im Potpourri popularisiert werden. Bei unvoreingenommener Betrachtung könnte man sogar die Aneinanderreihung solcher »klassischen « Zitate euphemistisch als eine spezielle Ausprägung des Komponisten-Kanons bezeichnen, mit welchem man Franz Schubert seine Reverenz erweist und seinen Ruhm in alle sozialen Schichten trägt - so äußerte sich jedenfalls der musikalische Schöpfer des Singspiels Dreimäderlhaus, Heinrich Berté (Schneidereit 1964: 390).

Intellektuelle Kritiker möchten sich allerdings des Potpourris als lästigem Komplement der Kunst entledigen, indem sie es entwerten und damit aus den Kreisen der Kunst zu verbannen suchen... 


\section{Normenverletzungen im Schubert-Potpourri}

Daher ist ein Nachdenken darüber angebracht, warum die bürgerliche Kunstkritik sich von Versuchen zur Popularisierung bedeutender Komponisten provoziert fühlt und sie als Verletzungsakte verbucht (GiesbrechtSchutte 2001). Die soziale Funktion des Potpourris spielt dabei keine Rolle, argumentiert wird nur auf der Basis ästhetischer Normen und formaler Kriterien, nach denen die Qualität des Potpourris zweifelhaft erscheint.

Es entfällt, wie das Schubert-Beispiel zeigt, ein durchdachtes Formkonzept, das auf Symmetrien, Analogien und auf einem Bezugssystem beruht, in dem die Einfälle des Komponisten miteinander agieren und sich entfalten können. Dafür gibt es viele geborgte »Themen«, die kaum in Beziehung zueinander treten, ungleich lang sind und daher zu unübersichtlichen Proportionen führen. So darf die Bühnenmusik zu Rosamunde (2) das gesamte erste Drittel des Stückes besetzen und in einer A-B-A-Form auftreten, die im Rahmen des Potpourris im wahrsten Sinne des Wortes »aus der Reihe tanzt«.

Die übrigen Zitate aus der Klaviermusik oder dem Sololied sind in ihrem Umfang eher einander angeglichen. Der Bearbeiter strebt, wo es möglich ist, die Nähe zum Originalklang an, indem er streckenweise notengetreu zitiert. So übernimmt er Hauptteil (4) und Trio (9) des Militärmarsches, den Sehnsuchtswalzer (3) und den Deutschen Tanz (5) in der gesamten Länge, wenn auch ohne Wiederholungen, und erweckt so den Eindruck der Authentizität. Wie ein Original wirkt auch das Lied Ungeduld (8), das sich immerhin in seiner vollständigen, mit Text versehenen ersten Strophe zeigt.

Vollkommen anders liegt der Fall bei dem Zitat aus der 8. Sinfonie, einer Gattung, die in der ästhetischen Hierarchie an oberster Stelle rangiert. Hier kann von Authentizität keine Rede mehr sein, denn vom sinfonischen Zusammenhang bleibt nur ein winziger, wenn auch charakteristischer Ausschnitt (7) im Potpourri zurück. Das einprägsame Thema ist der klanglich verfremdete Repräsentant der ausgesperrten sinfonischen Großform, die nur noch als Steinbruch für thematisches Material, in diesem Fall für eine melancholische Liedweise, eine Rolle spielt.

Hier kann die Kritik wohl mit besonderem Nachdruck die Qualität des Potpourris bezweifeln. Herrschenden ästhetischen Normen zufolge verletzt jeder eigenmächtige Eingriff in die Struktur und jede Fragmentierung historischer Texte ihre Aura und führt zur Beschädigung des Originals. Im Potpourri ist die Fragmentierung total. Themen oder charakteristische Teilstücke aus der melodisch-rhythmischen Oberfläche eines Werkes werden zur Verfügungsmasse für Arrangeure. 
Diese Kritik ist allerdings nur in Bezug auf dominante ästhetische Normen relevant und durch Vergleiche fühlbar. Lässt man sich unbefangen auf das Schubert-Potpourri ein und spielt es in seiner ganzen Länge am Klavier durch, so vermisst man eigentlich nichts. Kennt man jedoch die Originale, verfügt über Klangvorstellungen und Erinnerungen an den jeweiligen Kontext, so stellt sich ein Gefühl des Verlustes ein, eine Art Phantomschmerz, wie nach einer Amputation.

Kenner vermissen vermutlich die im Original verzeichneten Wiederholungen, aber das fällt wohl noch am wenigsten ins Gewicht. Bei der Abtrennung sämtlicher Folge-Strophen im Sololied wird der Verlust schon eher empfunden, da die angegebene erste Strophe nach textlicher Weiterführung drängt. Das Auseinanderdriften von Hauptteil und Trio beim Militärmarsch (4 und 9) irritiert, wenn man weiß, dass nach dem Willen des Komponisten das Trio dem ersten Teil unmittelbar zu folgen hat und sich daran erinnert, wie die anschließende Wiederholung des Hauptteils dem Werk seine gewohnte befriedigende Abrundung gibt.

Eine unangenehme Überraschung auch für wenig erfahrene Hörerinnen und Hörer ist der plötzliche Abbruch einer melodischen Linie, wie er beim Zitat des Moment Musical (6) zu beobachten ist. Nach vollständiger Wiedergabe des achttaktigen Eröffnungsabschnitts in der Originaltonart f-Moll findet der nachfolgende Mittelteil in As-Dur ein vorzeitiges und gewaltsames Ende, um den Einstieg in das nachfolgende Sinfoniethema (7) vorzubereiten. Dieses Vorgehen lässt Zweifel an der handwerklichen Kompetenz des Bearbeiters aufkommen, denn die zur Überleitung umgeformte Melodie basiert auf einem wenig überzeugenden Kadenzvorgang und erfolgt an dieser Stelle zu Lasten des klassischen symmetrischen Formkonzeptes.

Den Höhepunkt erreichen die Verlustempfindungen beim Auftreten des berühmten zweiten Themas der 8 . Sinfonie, da seine ursprüngliche Umgebung, ein riesiges sinfonisches Terrain voller harmonischer Gegensätze und phantasievoller sozialer Beziehungen von Themen und Motiven abhanden gekommen ist. Übrig bleibt ein vollständig vereinsamtes Subjekt im Gewand eines Klaviersatzes, der hilflos versucht, etwas von dem ursprünglichen Zauber der Streicher-Melodie wiederzugeben.

Alle diese Erscheinungen sind Defizite nur im Verhältnis zu den Herkunftswerken. Wer sich im kanonisierten Repertoire nicht auskennt oder sich darüber hinweg setzt, für den ist das Potpourri ein im wahrsten Sinne »einfallsreiches « Spielstück, informativ und unterhaltend und mehr als die Summe seiner Teile, in denen der Komponist über das gesamte Stück hinweg thematisch anwesend ist. 


\section{Verlust der Arbeit}

Das Potpourri macht es dem Rezipienten absichtlich leicht. Es bedarf nur einer geringen intellektuellen Anstrengung, um die überschaubare Anzahl geschlossener musikalischer Gestalten wahrzunehmen, die in abwechslungsreicher Folge in Erscheinung treten. Die Überleitungen zwischen den einzelnen Abschnitten sind nur kurze Atempausen und bereiten den Hörer jeweils auf den Genuss neuer melodischer Einfälle vor. Man kann sie beim SchubertPotpourri kaum als kompositorische Eigenleistungen des Bearbeiters bezeichnen. ${ }^{9}$ Es verbleiben die subjektiven Einfälle des Komponisten, die gerade noch einen Schatten von Kunst zurücklassen. Theodor W. Adorno bezeichnet das Potpourri als »Surrogat musikalischer Form « und als - wenn auch ungeordnete und verwirrende - Überlebensmöglichkeit musikalischer Gedanken (Adorno 1964: 22-24).Was hier an Kunst erinnert, sind isolierte Themen, Schuberts zum Kranz gewundene »Blüten«, die schönsten und dekorativsten Teile der Pflanzen. Ihnen fehlen jedoch die Blätter, Stängel und Wurzeln. Die Pflanze zeigt sich - um beim Bild zu bleiben - beschädigt, aber dennoch nicht gesichtslos. Die Physiognomie des Komponisten ist an der Oberfläche noch erkennbar.

Es herrscht jedoch ein Mangel an gedanklicher Arbeit, es fehlt der ordnende Geist, der die Vielfalt der angebotenen Fragmente immer neu zu gestalten weiß. Es fehlt das für ein Werk konstitutive Moment der Einheit in der Mannigfaltigkeit, ein integrales Prinzip, das Sinn, Logik und die Idee eines planvoll organisierten Ganzen herstellt. Dieses wird durch kompositorische Arbeit erzielt, die beim Potpourri entfällt. Es kann nur mit der Aneinanderreihung geliehener Themen und Abschnitte aufwarten, die nicht ausreichend miteinander verknüpft sind. Die Idee eines musikalischen Kunstwerkes schließt aber den individuellen Einfall erst in Verbindung mit seiner Verarbeitung und Positionierung in einem größeren Zusammenhang ein, und Beziehungsreichtum wird ein wichtiger Maßstab für ästhetische Qualität. Die Form des Potpourris ist diesem Strukturkonzept geradezu diametral entgegengesetzt.

9 Insgesamt bilden die Übergänge nur einen Anteil von knapp 10\% (23 Takte) des Stückes. Der längste dauert sieben Takte und steuert nach einer Generalpause über Doppeldominante und diverse Dominantakkorde den Sehnsuchtswalzer (3) an. Die übrigen sind noch kürzer und arbeiten sich so rasch wie möglich über die Dominante zum nächsten Zitat vor. 


\section{Unvereinbarkeit von Arbeit und Unterhaltung}

Das Potpourri kommt in jeder Hinsicht mit einem geringen Aufwand an Arbeit aus. Auch Rezipienten müssen sich nicht mehr um mühevollen Mitvollzug bemühen, sondern können sich ohne Anstrengung an der Vielfalt der gebotenen Themen erfreuen. Die Arbeit des Komponisten, aus dessen Werk sich das Potpourri nährt, ist vergessen, als hätte es sie nie gegeben. Franz Schubert, der in Beethovens kompositorischen Künsten, vor allem in seiner ausgefeilten Satztechnik ein Vorbild sah, wird auf seine schönen melodischen Einfälle reduziert. Verschwunden sind Kontexte, originelle Gegenstimmen und Begleitfiguren sowie die genialen harmonischen Glanzlichter und alle Bemühungen des Komponisten um eine individuelle, unverwechselbare Werkgestalt. Mit dieser Arbeit räumt das Potpourri auf und setzt dafür eine durch den Namen Schuberts garantierte »kunstvolle« Unterhaltung an ihre Stelle.

Vielleicht ist es gerade die Missachtung der Arbeit, des ästhetisch-intellektuellen Diskurses, welche bürgerliche Kritiker des Potpourris so außer Fassung bringt. Das Wertsystem der Kunst ist empfindlich verletzt, wenn die geistige Arbeit entfällt.

Wie wohl kaum eine andere gesellschaftliche Gruppe bezieht das Bildungsbürgertum sein Selbstwertgefühl aus der Arbeit. Ludwig van Beethoven ist nicht zuletzt deshalb als eines der bedeutendsten Genies und Vertreter höchsten Geistesadels verehrt worden, weil sein Werk die Spuren intellektueller Anstrengung besonders deutlich wiedergibt, wie seine Skizzenbücher zeigen. Arbeit wird als bürgerlicher Wertbegriff gegen Müßiggang, geerbten Reichtum, Genuss und bloße Unterhaltung ins Feld geführt. Arbeit bedeutet die Vervollkommnung der Individualität (Ueding 1973: 25) und enthält die Möglichkeit des sozialen Aufstiegs. Was sich durch Arbeit erschließen lässt, ist wertvoll. Eine Musik, in der die Arbeit erkennbar keine wichtige Rolle spielt, kann daher im bürgerlichen Wertsystem keinen Platz in den oberen Rängen beanspruchen. Diese sind vom Kanon großer Kunstwerke besetzt, die sich auch der bürgerlichen Elite nur durch geistige Mühen erschließen.

Die ästhetische Intoleranz gegenüber dem Potpourri scheint also auf der Verletzung dieser zentralen bürgerlichen Norm zu beruhen, nach der Arbeit missachtet und durch Momente der Unterhaltung ersetzt wird. 


\section{Moderne}

Der Typus des modernen Potpourris nutzt den Begriff Kunst fast nur noch zu Werbezwecken und vermarktet, was auf großstädtischen Bühnen oder im Tonfilm gerade erfolgreich ist, vorwiegend Schlager und Gesellschaftstänze sowie Opern-Arien und Operetten-Couplets. Aktualität und Innovation sind seine Maßstäbe und ein meist rasches Verfallsdatum sein Markenzeichen. Seine Schnelllebigkeit wird vom Publikum, von Produzenten und den am Vertrieb Beteiligten begrüßt und die Suche nach jeweils neuartigen musikalischen Reizen als Element des Fortschritts gewertet. Schlager-Potpourris mit ihrem oft chaotischen Nebeneinander unterschiedlicher Bühnen- und Tonfilm-Neuheiten bringen das ereignisreiche musikalische Großstadtleben ins bürgerliche Haus, wo sie neben populären Kunstformen oder anderen leichten Stücken in entsprechenden Notenbüchern angeboten werden, sodass für jeden Geschmack und jedes Bildungsniveau etwas dabei ist.

Mit innen kommt ein frischer Wind in die Salons. Die aktuelle Unterhaltungsmusik schließt eine gewisse Weltoffenheit ein, zumindest jedoch die Bereitschaft, sich auf rasche Wechsel einzulassen und sich ohne Zögern für die neuste musikalische Mode oder exotische Tanzformen zu begeistern. Das Zuhören erfordert wenig Anstrengung. Gefragt sind eher Fähigkeiten zum Mitmachen sowie Freude an sinnlichen Eindrücken und Späßen, etwa an humorigen Texten oder aufregenden synkopierten Rhythmen.

Genüsse dieser Art bieten zeitgenössische Schlager und - in komprimierter Form - die Schlager-Potpourris. Da sie immer nur die Kennmelodie, die Strophe oder den einprägsamen Refrain wiedergeben, lassen sich zahlreiche Neuheiten aus Film, Radio und Bühne in einem einzigen Exemplar unterbringen. Manche Beispiele aus den Musikalischen Edelsteinen enthalten ganze Listen aktueller Schlager, so z.B. ein Wunsch-Programm 1931. Das neue Schlagerpotpourri von Gerhard Mohr (XIV, 1931). In buntem Reigen erscheinen hier zehn verschiedene Foxtrott-, Tango-, Marsch- und OnestepMelodien, eingebettet in relativ umfängliche Vor-, Nach- und Zwischenspiele. Sie stammen zum Teil aus Tonfilmen oder sind aktuellen Notenausgaben entnommen und bieten, wie der Titel des Potpourris behauptet, die am meisten nachgefragten Schlagermelodien eines ganzen Jahres an.

Auf Grund solcher Beispiele ist man versucht, von einem inoffiziellen Unterhaltungskanon zu sprechen, der ein Ensemble der beliebtesten Schlagermelodien präsentiert, Modell bildend ist und den Geschmack der Zeit bestimmt. Das in seiner Zusammensetzung stets wechselnde, von Novitäten überquellende Schlager-Potpourri erfüllt zumindest die Funktion eines Kom- 
pendiums von Neuerscheinungen der Schlager-Branche und ist im Gegensatz zum klassischen Kanon, der weiterhin Gültigkeit besitzt, fortlaufend Veränderungen ausgesetzt.

\section{Aktuelle Potpourris: 1908 bis 1939}

Der rasche Wandel der Sujets im Potpourri ermöglicht einen Einblick in die jeweils aktuelle Mode der Zeit, die mit Begriffen wie Unterhaltung und Lebensstil nicht immer ausreichend zu erfassen ist. Ästhetische Präferenzen gehen darin eine Verbindung mit dem »Zeitgeist « der Jahre 1908 bis 1939 ein, in denen drei verschiedene Herrschaftsformen zu verkraften sind. Selbst in der bescheidenen Gestalt des Potpourris lassen sich - ebenso wie im gesamten Repertoire der Musikalischen Edelsteine - Spuren davon auffinden.

Eine optimistische Stimmung äußert sich in humoristischen Varianten der Vorkriegsjahre. In Band I gibt es eine eigene Rubrik »Gelegenheitsmusik und Humoristika«, zu der auch das humoristische Potpourri mit dem Titel Fidelitas, ein Op. 40 von John Klein, gehört. 21 verschiedene Couplets und Lieder aus zeitgenössischen Operetten - unter anderem Albert Lortzing: Der Waffenschmied, Paul Lincke: Frau Luna, Karl Millöcker: Der Bettelstudent - wechseln sich ab mit Gassenhauern wie »Komm Karlinchen!« oder »Ist denn kein Stuhl da für meine Hulda«. Auch die drei anderen Vorkriegsbände favorisieren diesen Typus. ${ }^{10}$

Der muntere Tonfall wird nach Beginn des Ersten Weltkrieges überlagert durch Beiträge aus Kriegs-Operetten, die dem »neuesten « Potpourri Der jüngste Jahrgang von Camillo Morena (Band VI) einige Propaganda-Elemente hinzufügen, ohne jedoch auf humoristische Untertöne zu verzichten. Das berühmte Couplet »Der Soldate, der Soldate ist der schönste Mann im Staate « aus der Operette Immer feste druff von Walter Kollo ist ein gutes Beispiel dafür. Kollo ist auch noch mit anderen Songs, unter anderem aus Extrablätter vertreten. Weitere Zitate stammen zum Beispiel aus Unsere Feldgrauen von Robert Winterberg sowie Kam'rad Männe und Woran wir denken von Jean Gilbert alias Max Winterfeld. Sie vervollständigen das von Morena zu einem »Op. 130« hochstilisierten »Werk«.

10 Bd. II: Humor-Potpourri über 18 Schlager mit unterlegtem Text von Oscar Fetrás, Op. 152, Obertitel: Im 7ten Himmel; Bd. III: Operetten-Revue. Humoristisches Potpourri mit überlegtem Text von Oscar Fetrás, Op. 171; Band IV: Berlin wackelt. Potpourri mit überlegtem Text von C. [amillo] Morena, Op. 123. 
Spätere Edelstein-Bände aus der Zeit der Weimarer Republik unterhalten ihr Publikum bis in die 1930er Jahre mit modernen Tanz- und Tonfilmschlagern, aber die Herausgeber sind ebenfalls bemüht, dem jugendbewegten Singen einen Platz einzuräumen, und präsentieren auch im Potpourri traditionelle Volkslieder. In Band XI (1927) ist ein Deutsches-VolksliederPotpourri von Oscar Fetrás abgedruckt, in dem Studenten- und Liebeslieder und zum Schluss das Deutschlandlied vorkommen, das seit 1922 deutsche Nationalhymne ist. Im Lieder-Potpourri Op. 76 Vom Rhein zur Donau von Max Rhode (XII, 1929) wird der Rhein gepriesen, der im patriotischen Diskurs der Zeit eine wichtige Rolle spielt. Es beginnt und endet mit so bekannten Weisen wie »Warum ist es am Rhein so schön« und »Das Herz am Rhein«. Dazwischen wird man über weitere regionale deutsche »Volksweisen « bis hin nach Österreich geführt und mit populären Wiener Liedern von Robert Stolz, Ralph Benatzky und Ernst Arnold ${ }^{11}$ bekannt gemacht. Der Zeitpunkt des Erscheinens erlaubt die Vermutung, dass hier auf dem Feld der Kultur der Zusammenschluss mit Österreich geprobt wird.

Seit 1930 kommen martialische Töne hinzu. In Band XIII wird ein Marschlieder-Potpourri mit dem Titel Jung-Deutschland angeboten. Das Arrangement von Leopold Weninger enthält 18 bekannte Soldatenlieder wie »In der Heimat gibt's ein Wiedersehn«, »Drei Lilien« oder »Lippe-Detmold« und endet wieder mit dem Deutschlandlied.

»Volks- und Stimmungslieder im Walzertakt « ${ }^{12}$ sind eine in der heraufziehenden NS-Zeit geschätzte Alternative zu Schlagern. Konkretere Spuren der NS-Herrschaft lassen sich jedoch in den letzten Bänden der Musikalischen Edelsteine nicht finden. Die Reihe wird mit Band 17 (1939) beendet, der mit dem Potpourri Singende Soldaten und mit Liedern wie "Schwarzbraun ist die Haselnuss « oder »Erika«, dem rhythmisch markanten, im Zweiten Weltkrieg überaus populär gewordenen Marschlied von Herms Niel, auf den herannahenden Zweiten Weltkrieg verweist.

Dieser Überblick über nur etwa 30 Jahre soll zeigen, in welchen kurzen Zeiträumen sich das Unterhaltungs-Repertoire neu orientiert und wie auch das Potpourri sich allem, was gerade aktuell, politisch erwünscht und populär ist, öffnet. Damit wird es vielgestaltiger und erfüllt erkennbar die Kriterien der Moderne.

11 Robert Stolz: »Im Prater blüh'n wieder die Bäume«, »Der Frühling in Wien«, "Wien wird bei Nacht erst schön«; Ralph Benatzky: "Ich muß wieder einmal in Grinzing sein«; Ernst Arnold: »Da draußen in der Wachau«.

12 Band XIV: Zurück zum Walzer. Potpourri aus beliebten Volks- und Stimmungsliedern im Walzertakt, Op. 20, von Josef Freudenthal mit Beispielen wie »Du, du liegst mir im Herzen«, »Es war einmal ein treuer Husar «, »O du wunderschöner deutscher Rhein«, »Waldeslust«u.a. 
Alle Potpourris in der Sammlung Musikalische Edelsteine fassen in sich zusammen, was eine Zeit auf dem Gebiet der Musik für zentral und mitteilenswert hält. Insofern ist zumindest formal eine gewisse Verwandtschaft zur Struktur eines Kanons vorhanden.

\section{Schluss}

Die Fähigkeit des Potpourris, diverse Musikarten in sich aufzunehmen, macht es zu einem Prototyp der Moderne des 20. Jahrhunderts, zu deren Wesensmerkmalen ein radikaler Wandel in Fragen des Geschmacks und eine bisher nicht gekannte Pluralität musikalischer Erscheinungsformen gehören. Charakteristisch für die Zeit ist, dass herrschende ästhetische Normen an Einfluss verlieren und der Konkurrenz unterschiedlicher Stile, Gattungen und ästhetischen Konzepte Tür und Tor geöffnet werden. Im Potpourri vereinen sich diese konträren Bestrebungen, denn es kann ebenso an bedeutenden Kunstwerken wie an der aktuellen Unterhaltungsmusik partizipieren. Die immer gleiche Binnenstruktur - die Aufeinanderfolge von Highlights einer Oper oder einer Schlagersaison - stellt Unterhaltung und sinnliches Vergnügen auf die gleiche Stufe wie Bildung und Kunst. Auf diese Weise ist das Potpourri verschiedenartigen Interessen und Bildungsniveaus zugänglich, und dieses Spektrum der Bezugsmöglichkeiten sichert seinen Erfolg im bürgerlichen Musikleben. Zugleich eröffnet es Perspektiven für einen liberalen Begriff von Kultur, in dem Kunst und Unterhaltung in ihren unterschiedlichen Abstufungen gleichermaßen ihren Platz finden müssen.

Dennoch hat das Potpourri mit seinem mangelhaften ästhetischen Ruf zu kämpfen. Seine Abhängigkeit von fremden Texten weckt nicht nur Interessen, sondern zieht auch den kritischen und exklusiven Vergleich mit den Originalen nach sich. Die Messlatte ist das kanonisierte Repertoire, das Ergebnis eines Auswahlprozesses, der ästhetische Höchstleistungen würdigt, mustergültige Kunstwerke idealisiert und geniale Komponisten auszeichnet. Gemessen an diesem Kanon sinkt der Wert des Potpourris, ist aber innerhalb ästhetischer Bezugsysteme dennoch untrennbar mit den Normen der Kunst verbunden.

Anders fällt das Ergebnis aus, wenn es nicht um den künstlerischen Wert, sondern um die soziale Funktion des Potpourris geht und seine bereits in der Struktur angelegten komplementären Qualitäten erkennbar werden. Es weist über sich selbst hinaus auf das gesamte bürgerliche Musikleben, auf den Bereich der Kunst ebenso wie auf den der Unterhaltung. Es verkraftet als bürgerliche Hausmusik die Spaltung zwischen Kennern und Liebhabern 
und wirbt für das Interesse an bedeutenden Komponisten. Es negiert existierende Rangordnungen zwischen musikalischen Formen und Gattungen und verbindet Kunst und Leben, indem es Einfluss nimmt auf die Gestaltung bürgerlicher Lebens- und Kommunikationsformen der Zeit.

\section{Literaturverzeichnis}

Adorno, Theodor W. (1964). "Schubert« [1928]. In: Ders., Moments musicaux. Neu gedruckte Aufsätze 1928-1962. Frankfurt/M.: Suhrkamp, S. 18-36.

Ballstedt, Andreas (1997). »Potpourri. «In: Die Musik in Geschichte und Gegenwart. Sachteil, Bd. 7. Hg. v. Ludwig Finscher. Kassel, Stuttgart: Bärenreiter/Metzler (2. Aufl.), Sp. $1760 f$.

Dahlhaus, Carl (1972) »Über die >mittlere Musik des 19. Jahrhunderts. «In: Das Triviale in Literatur, Musik und bildender Kunst. Hg. v. Helga de la Motte-Haber. Frankfurt/M.: Vittorio Klostermann, S. 131-147.

Engel, Hans (1962). »Potpourri.« In: Die Musik in Geschichte und Gegenwart. Bd. 10. Hg. v. Friedrich Blume. Kassel: Bärenreiter (1. Aufl.), Sp. 1720.

Geuen, Heinz (1995). »>Das hat die Welt noch nicht gesehen und Revue als Embleme populärer Kultur der 20er Jahre, « In: Es liegt in der Luft was Idiotisches. Populäre Musik zur Zeit der Weimarer Republik. Hg. v. Helmut Rösing (= Beiträge zur Popularmusikforschung 15/16). Baden-Baden: Coda, S. 5268.

Giesbrecht, Sabine (2000): "Daisy oder Sylvia - Kunst unter der Optik des Lebens. « In: Frauen- und Männerbilder in der Musik. Festschrift für Eva Rieger zum 60. Geburtstag. Hg. v. Freia Hoffmann, Jane Bowers und Ruth Heckmann. Oldenburg: Bibliotheks- und Informationssystem der Universität, S. 97-110.

Giesbrecht-Schutte, Sabine (2001): »-Klagen eines Troubadours-. Zur Popularisierung Schuberts im Dreimäderlhaus. In: Martin Geck. Festschrift zum 65. Geburtstag. Hg. v. Ares Rolf und Ulrich Tadday. Dortmund: Klangfarben Musikverlag, S. 109-133.

Hilmar, Ernst / Jestremsky, Margret (Hg.) (1997). Schubert-Lexikon. Graz: Akademische Druck- und Verlagsanstalt.

Schneidereit, Otto (1964). Operettenbuch. Berlin (DDR): Henschelverlag (10. Aufl.).

Schumann, Robert (1889). Gesammelte Schriften über Musik und Musiker. Bd. 2. Hg. v. Heinrich Simon. Leipzig: Reclam.

Storck, Karl (1911). "Gartenkonzerte«. In: Ders., Musik-Politik. Beiträge zur Reform unseres Musiklebens. Stuttgart: Greiner \& Pfeiffer, S. 102.

Ueding, Gert (1973). Glanzvolles Elend. Versuch über Kitsch und Kolportage. Frankfurt/M: Suhrkamp. 


\begin{abstract}
Musikalische Edelsteine, a series of collections of arrangements for the piano published between 1908 and 1939, contain various forms of potpourris. These potpourris, arranged from melodies from operas, operettas, popular songs and dances as well as rather sophisticated works of art music, belong to a sphere between art and the popular. Middle-class families accepted potpourris, e.g. with subjects from compositions by Franz Schubert, as part of their social life, whereas bourgeois critics judged them by categories of art, criticizing the disruption of the artist's work, but, nonetheless, justifying the medley for its educational values in teaching works of the classical canon to people of lower education. Potpourris with pieces of operettas, with dances or popular songs are significant for the modern times at the beginning of the 20th century.
\end{abstract}

